\title{
Subject Index Vol. 33, 1996
}

Acetylcholine 66, 182 Acetylcholinest erase 182 Acute episode 169 Additivity $100 \alpha$-, ß-Adrenoceptors 147 Adrenocorticotrop(h)ic hormone 71,173

- 4-9 90 Affective disorder 55 Aging 210 Agoraphobia 118 Alcohol preference 85 Amantadine 80 Amitriptyline 155 Amygdala 189 Analysis of variance 100 Animal model(s) 85

-, depression 147 Anticholinergics 80 Antidepressant(s) 55, 66 Anxiety 118,189 L-Arginine 76 Arousal 173

Artificial neural networks 106,155 Auditory-evoked potential(s) 90,113,164

Baseline correction 100 Behavior 85, 182 Behavioral sensítization 21 Brain dialysis 132

- $\quad$ electric source analysis 90

Brainstem 164

Brofaromine 160

Bulimia nervosa 71

$\mathrm{Ca} 2+66$

antagonists 55 Calcium 169 Catecholamines 173 Cerebellum, rat 60 Cerebral activation 127

blood flow 127

metabolic rate

metabolism 127 Cerebrospinal fluid 169 Choline acetyltransferase 182 Circadian rhythms 71 Citalopram 17 Clomipramine 17,147 Cognitive functions 48 
Conditioned response 21 Continuous Performance Test 127 Corticotropin-releasing hormone 90 Cortisol 71, 173 Cultured neurons 66 Defence 173 Dementia 106 18F-Deoxyglucose 1 Descriptive data analysis 32,160 2,3-Dichloro- $\alpha$-methylbenzylamine

132 Digital mobile radio telephone 41 Distance computation 32 Dopaminergic dominance 21

EEG 138,210

mapping 32

Electroconvulsive therapy 138

Electrocorticogram 100

Electrodermal activity 147

Electromagnetic fields 41

Elevated T-maze 189

Emotions 173-

B-Endorphin 71, 173

Epinephrine 132

Ethanol 60

Event-related potentials 48

Fear 173 Frontal cortex 66 lobe 1

Galactorrhea 142

Generalized anxiety disorder 196

Geriatrics 12

Glucose 1

Glutamatergic system 80

Growth hormone 132, 173

Haloperidol 12,76, 186 Heritability 113 HighK+ 66 Homoscedasticity 100 [3/4]PK11195 28 Hypofrontality 127 Hypothalamic-pituitary-adrenocortic system 90 Hysterical neurosis 186 
Infectious mononucleosis 202 Inferior cervical ganglion 147 Inhibition 76 Inositol 1,3,4,5-tetrakisphosphate 60

1,4,5-trisphosphate 60 - - receptor 60 Intracellular Ca2+mobilization 55

Learning ability 182 Likelihood profile 113 Lithium 17

Locomotor activity 132 Locus ceruleus 164 Lundby study 118

Magnesium 169 Major depression 48 MAO A inhibitor 160 Mean power spectrum 100 Memantine 32 Memory 189 Methamphetamine 21 3-Methoxy-4hydroxyphenylglycol

132 Moclobemide 48 Mood 202 Mortality 118 Mouse brain 182

Multiple discriminant function 106 Muscarinic receptors 147

NADPH 76

Neural circuits 1 Neuroleptic parkinsonism 80 Neuroleptics 142 Neuropsychiatry 97 Nicotine 210 Noradrenaline 66 Normal population 118 Normality 100 NOS 76

Obsessive-compulsive disorder 97 Oxyhemoglobin 76

P45O 76

Panic disorder 118,164 Performance 202 Peripheral-type benzodiazepine receptor 28 
Pharmaco-EEG 32, 155, 160

Pharmacotherapy 196

Phenylethanolamine-N-methyltrans-ferase inhibitors 132

Plasma levels 12

Positron emission tomography 1

Potentiation, antidepressant treatment 17

Prolactin 173, 186

Prospective study 118

Quantitative EEG 97, 106, 155

- $\quad$ - profile 48

Rat 100

- $\quad$ C6BU-lglioma cells 55

Reciprocal balance 21

Reduced haloperidol 12

Remission 169

REM sleep 41, 155

Schizophrenia 127,142, 169

Scopolamine 21

Serotonergic receptors 17

Serotonin 17,55, 189

Serum 169

Side effects 80

Single-subject experiments 196

Skin innervation 147

Sleep EEG 41

Smoking 210

Spectral analysis 97,160

power density 41

Stereotyped behavior 21

Sulpiride 186

Superior cervical ganglion 147 Swim stress 28

Tetrahydrobiopterin 85 Thalamus 1

Therapeutic response 138 Tourette syndrome 97 Transformation 100 Twins, monozygotic and dizygotic 113

Ultraviolet A 182

Verapamil 55 Vigilance model 32 